\title{
CLÍNICA ANALÍTICA DE LAS ORGANIZACIONES: UNA PROPUESTA CONCEPTUAL
}

\author{
Clínica analítica das organizações: uma proposta conceitual \\ Analytical clinic of organizations: a conceptual proposal
}

Clinique analytique des organisations: une proposition conceptuelle

\begin{abstract}
Horacio Manrique ${ }^{1}$
Psicólogo, Magíster en Filosofía y Doctor en Psicología. Profesor en la Universidad EAFIT (Medellín, Colombia). Departamento de Psicología. Departamento de Organización y Gerencia.
\end{abstract}

Isabel Cristina Lopera ${ }^{2}$

Psicóloga, Magíster en Desarrollo Humano Organizacional. Profesora en la Universidad EAFIT (Medellín, Colombia). Departamento de Psicología. Departamento de Organización y Gerencia.

\begin{abstract}
Juan David Pérez ${ }^{3}$
Administrador de empresas, Magíster en Desarrollo Humano Organizacional. Profesor en la Universidad EAFIT

(Medellín, Colombia). Departamento de Psicología. Departamento de Organización y Gerencia.
\end{abstract}

\begin{abstract}
Resumen
Como producto de una investigación cualitativa de tipo hermenéutico, se presenta una propuesta para la aplicación en las organizaciones del método clínico. La base de este trabajo es un fundamento teórico en el cual se revisan algunos conceptos clásicos que sustentan la posibilidad de articular los campos clínico y organizacional. El método clínico-analítico no es exclusivo del ámbito de la salud y la enfermedad, por el contrario, ha sido utilizado en campos tan diversos como el derecho, la sociología, la pedagogía, entre otros. Este método consiste en la operación de descomposición de discursos en sus elementos constitutivos. Si se acepta que las organizaciones son concreciones de discursos fundantes (instituciones), se aceptará que el método clínico-analítico es pertinente para su estudio e intervención. Además, se argumenta que también el ser humano está constituido por instituciones. Aceptando estas premisas, se puede hablar de una gestión humana de orientación analítica, cuya esencia son los espacios de verbalización individuales y grupales, basados en una filosofía de gestión integradora. Como consecuencia, se propone que esta gestión humana de orientación analítica propende al desarrollo humano individual, grupal y organizacional.
\end{abstract}

Palabras Clave: Método clínico; Método analítico; Organización; Concepción de hombre; Gestión humana.

\section{Resumo}

Como resultado de um estudo qualitativo do tipo hermenêutico, apresenta-se uma proposta para implementação em organizações de um método clínico. A base deste trabalho é uma base teórica na que alguns conceitos clássicos subjacentes a capacidade de articular os campos clínicos e organizacionais são revistos. O método clínico-analítico não é exclusivo para o campo da saúde e da doença, por outro lado, tem sido utilizado em campos tão diversos como direito, sociologia, pedagogia, entre outros. Este método envolve a operação de descomposição de discursos nos seus elementos constituintes. Aceita-se que as organizações são concreções de discursos fundantes (instituições), ou seja, aceita-se que o método clínico-analítico é relevante para o estudo e intervenção. Além disso, argumenta-se também que os seres humanos se constituem nas instituições. Aceitar estas premissas, implica que

\footnotetext{
1 hmanriqu@eafit.edu.co

2 ilopera@eafit.edu.co

3 jperezp3@eafit.edu.co
} 
pode-se falar de um gerenciamento do ser humano de orientação analítica, cujos espaços de verbalização individual e grupal são essenciais, baseados em uma filosofia de gestão integrada. Como um resultado disso, propõe-se que esta gestão humana tende a ser analiticamente orientada ao indivíduo, ao grupo e ao desenvolvimento humano organizacional em geral.

Palavras chave: Método clínico; Método analítico; Organização; Concepção do homem; Gestão humana.

\begin{abstract}
As a result of a qualitative study of hermeneutical type, we present a proposal for implementation in organizations of the clinical method. The basis of this work is a theoretical foundation on which we review some classic concepts underlying the ability to articulate the clinical and organizational fields. The clinical-analytical method is not exclusive to health and disease issues; conversely, it has been used in such dive rse fields as law, sociology, pedagogy, among others. This method involves the decomposition of discourse into its constituent elements. If it is accepted that organizations are concretions of founding speeches (institutions) it will be accepted that the clinical-analytical method is relevant for its study and intervention. Furthermore, it is argued that also humans are constituted by institutions. Accepting these premises, it is possible to speak of a human management analytically orientated, based on a philosophy of integrative management, that provides spaces for individual and group verbalization. As a consequence, it is proposed that this human management of analytically orientated tends to enhance the development of human beings, groups and the organization itself.
\end{abstract}

Key Words: Clinical method; Analytical method; Organization; Conception of human; Human resources management.

\title{
Résumé
}

En conséquence d'une étude qualitative du type herméneutique, une proposition de mise en œuvre dans les organisations de la méthode clinique est présenté. La base de ce travail est une base théorique dans laquelle certains concepts classiques qui sous-tendent la capacité à articuler les domaines cliniques et organisationnels sont passés en revue. La méthode clinique analytique n'est pas unique dans le domaine de la santé et de la maladie, d'autre part a été utilisée dans des domaines aussi divers que le droit, la sociologie, la pédagogie, entre autres. Cette méthode implique des discours d'opération de décomposition en ses éléments constitutifs. Si l'on admet que les organisations sont des concrétions de discours fondateurs (institutions), il sera admis que la méthode clinique d'analyse est pertinente pour l'étude et l'intervention. En outre, il fait valoir que les humains aussi consiste institutions. L'acceptation de ces locaux, on peut parler d'un être humain la gestion axée sur analytiquement, dont les espaces sont essentiellement individuelles et collectives verbalisation, basé sur une philosophie de gestion intégrée. En conséquence, il est proposé que cette gestion humaine tend à orientée analytiquement individuelle, de groupe et le développement humain de l'organisation.

Mots-clés: Méthode clinique; Méthode d'analyse; L'organisation; La conception de l'homme; La gestion humaine.

\section{Introducción}

La investigación de la cual se deriva este artículo teórico surgió a partir de la inquietud de algunos integrantes del grupo de investigación, acerca de la aplicación del método clínico en ámbitos diferentes al de la salud-enfermedad al cual ha estado asociado tradicionalmente (Bedoya y Schnitter, 2010). En efecto, desde las diversas perspectivas de los integrantes del grupo, se conjeturaba que dicho método tenía gran potencial para aplicarse en campos como el organizacional, lo cual fue convalidado posteriormente durante la investigación con datos teóricos y empíricos.

En el sentido teórico, son conocidas ya las propuestas de la sociología clínica norteamericana (Jaeckel, 1991), el análisis institucional, con su vertiente de la clínica de las instituciones (Foladori, 2008; Lapassade y Lourau, 1973; Schvarstein, 1998) y las clínicas del trabajo, de vertiente francesa y brasilera principalmente (Bendassolli y Soboll, 2011; Bermúdez, 2013; Orejuela, 2018). 
Entre otros nombres, según Fritz (1991), el surgimiento de la sociología clínica está unido a Ernest W. Burgess, sociólogo graduado de la Universidad de Chicago, que impartió sus cursos de sociología clínica entre 1928 y 1933. También está unido a Milton Winternitz, decano de la Escuela de medicina de la Universidad de Yale, que en 1929 impulsó la creación de una sección de sociología clínica. El primer artículo titulado Clinical Sociology, fue publicado en 1931 por Louis Wirth (de la Universidad de Chicago) (cf. Wirth, 1931). En 1978 se fundó la Clinical Sociology Association. En 1982 se fundó la Clinical Sociology Review (Jaeckel, 1991). Para Nicole Aubert y Vincent de Gaulejac (1993; cf. Gaulejac, Rodríguez y Taracena, 2006), la sociología clínica es un "campo interdisciplinario, tendiente al análisis de los fenómenos psicosociales en la relación hombre-trabajo" (Manrique, Lopera, Pérez, Ramírez y Henao, 2016, p. 23). Desde esta perspectiva, la sociología clínica implica: "1) la discusión crítica de comportamiento sociales espontáneos con observadores prácticos, 2) mediante el uso de datos clínicos disponibles y 3) con la participación directa en situaciones clínicas" (Fritz, 1991, p. 16, traducción nuestra). Según Fritz (1991), esta definición implica tanto el análisis de situaciones sociales como la intervención. El análisis clínico, dice, es la evaluación crítica de creencias, políticas y prácticas para la comprensión y mejora de una situación. La intervención se basa en el análisis y creación de nuevos sistemas, así como el cambio de los existentes. Además, implica una aproximación científica, humanista e interdisciplinaria y la focalización en un nivel (bien sea individual, interpersonal, grupal, organizacional, comunitario o internacional), pero integrando los otros niveles, y teniendo como punto de referencia un marco de trabajo sociológico.
En cuanto al análisis institucional, Lapassade y Lourau (1973) proponen una teoría, y a la vez una práctica, que busca modificar lo instituido (lo ya cristalizado en la organización) a partir de lo instituyente (la fuerza que posibilita el cambio), mediados por analizadores. Esto es, dispositivos que permiten, a partir del análisis (llevado a cabo entre el analista y los intervinientes), develar aquello que está siendo problemático en una organización. Es fundamental aquí la idea de autogestión o autoanálisis, de manera que el analista posibilita que las personas intervinientes sean quienes analicen $y$ develen sus situación grupal y afectiva, y al mismo tiempo propongan soluciones. Foladori (2008) retoma diversas propuestas del análisis institucional en sentido amplio (Lourau, Mendel, PichónRiviere, Bleger, Deleuze, Guattari, Castoriadis, entre otros), y propone una integración que propenda a una clínica de las instituciones en la que son fundamentales el vector político y el vector de la subjetividad, que se analizan a la luz de lo que denomina la fisura en lo instituido, que posibilita que emerja lo instituyente, pero a la vez, que lo instituido se percate de la fuerza instituyente y se recomponga con el fin de evitar el cambio.

Respecto a las clínicas del trabajo (Bendassolli y Soboll, 2011), concepto que propone Yves Clot e impulsa Dominique Lhuilier, tiene diferentes vertientes: clínica de la acción, de Gerard Mendel, clínica de la actividad, de Yves Clot y Daniel Faita, psicodinámica del trabajo, Cristophe Dejours, ergología, de Yves Schwartz, y la sociología clínica, también llamada psicosociología o psicología social clínica de Eugène Enriquez y Vincent de Gaulejac. Aunque cada una de estas vertientes tiene sus peculiaridades, debido a limitaciones de espacio y propósito en este artículo, en términos generales diremos que se caracterizan todas por el análisis teórico y práctico del placer y el sufrimiento del ser 
humano en su trabajo, desde una perspectiva cualitativa en la que lo fundamental la perspectiva en primera persona de cada sujeto en su actividad.

Bendassolli y Soboll (2011) y Malvezzi (2012a) plantean que está surgiendo un nuevo campo interdisciplinario que tiene lugar en la intersección entre la psicología organizacional la psicología clínica y del trabajo. En términos generales, estas perspectivas se preguntan por el lugar de la subjetividad con respecto a la organización y la institución, desde una perspectiva clínica. En una vía similar, pero con algunos conceptos novedosos retomados de Ramírez (2012; Ramírez, Lopera, Zuluaga y Ortiz, 2017), hacemos una propuesta conceptual de una clínica de las organizaciones, enmarcada en tres aspectos básicos: una concepción particular del método clínico-analítico, una concepción particular la organización y la institución y una conceptualización del sujeto como animal verbal.

Cabe aclarar que hablamos de una clínica de las organizaciones y no sólo de una clínica en las organizaciones, puesto que nuestra propuesta busca aportar conceptos que permitan tanto una lectura clínica de la organización desde lo universal, lo particular, lo singular e, incluso, lo peculiar, como una intervención en estos mismos niveles. De manera pues, que es una propuesta dialéctica, de intermodificación entre la teoría y la práctica (aquí sólo presentamos un resumen la parte conceptual; para otros aspectos, consultar: Henao, 2016; Manrique y otros, 2016; Pérez y Lopera, y 2016; Ramírez y Manrique, 2016). Entonces, en un primer momento, exponemos en qué consiste el método analítico, en tanto método de análisis del discurso. Continuamos con el método clínico, entendido como un método para el cuidado de lo singular y planteamos su articulación en el método clínicoanalítico. Pasamos luego a explicar nuestra idea de organización entendida como una concreción de discursos fundantes (instituciones). Por ello, es posible aplicar el método clínico-analítico en una organización dado que se aplica a lo que las constituye: discursos fundamentes. A continuación, presentamos la relación que existe entre la organización y el hombre en tanto animal verbal constituido, a su vez, por discursos culturales. Continuamos mostrando las implicaciones que estas concepciones tienen para el desarrollo humano. Finalmente, planteamos que el desarrollo humano sólo es posible si en la organización existe una filosofía de gestión y una gestión humana que lo respalden. Por último, resumimos nuestra propuesta conceptual sobre una clínica analítica de las organizaciones.

\section{Método analítico}

El método analítico es un método científico, es decir, un proceder basado en la "contrastación dialéctica entre la teoría y la práctica" (Ramírez, 1991; cfr. Lopera, Ramírez, Zuluaga y Ortiz, 2010a). El análisis es la descomposición de un todo en sus aspectos elementales con el fin de conocerlo y comprenderlo. Es posible analizar diferentes aspectos de la realidad tal y como lo muestran diversas disciplinas que utilizan el análisis como método fundamental tales como la química, la física, la geometría, la lógica, la biología, la sociología, la historia, la psicología, la filosofía, el psicoanálisis, entre otras. Para efectos de la presente investigación, asumimos que el método analítico es la aplicación del método científico a un discurso. Esto es, el método analítico tal y como lo concebimos, es la aplicación del análisis a un discurso (Ramírez, 1991; Lopera y otros, 2010a). Un discurso es "toda expresión de una estructura subjetiva" (Lopera y otros, 2010a, p. 14) y es a su vez una versión particular del logos entendido como principio universal 
articulante (cfr. Ramírez, 2012, ens. 49; Lopera, Manrique, Zuluaga y Ortiz, 2010b; cfr. Polanyi, 2005). Si aceptamos esta definición, no solo existen discursos hablados $\mathrm{o}$ escritos (formalizados en palabras), sino también discursos como los constituidos por los gestos de una persona, las obras de arte, el diseño de los espacios arquitectónicos, entre otros.

Método es una palabra que indica un modo o manera para investigar la verdad. En la antigua Grecia el método hacía parte del carácter del investigador que incorporaba dicha forma de proceder como un hábito que se convertía en parte de su forma de ser y vivir (Lopera y otros, 2010a; Manrique, 2008). De esta manera, lo epistemológico y lo ético son aspectos de un mismo proceso. Esta primera forma de entender el método, que se conserva hasta hoy, la denominamos el método como actitud (Lopera y otros, 2010a). Pero existe otra versión del método. Con el positivismo, el método se convirtió en una serie de pasos para llegar a un resultado. Es el método como algoritmo, concebido al estilo de una receta que puede seguirse mecánicamente (Lopera y otros, 2010a).

Desde mediados del siglo XX se ha dado un interesante debate acerca de los métodos o formas de razonar explícitas y mecánicas (método como algoritmo) y las formas de razonar tácitas y naturales (método como actitud) (cfr. Polanyi, 1967, 2005; Ryle, 1949/2005), especialmente en el campo de la toma de decisiones (cfr. Kahneman \& Klein, 2009; Klein, 2008; Gigerenzer, 2007; Taleb, 2006; Grove, 2005; Ross, Klein, Thunholm, Schmitt \& Baxter, 2004) y en el campo de la gestión del conocimiento organizacional (Nonaka $\&$ Teece, 2001).

Nuestra perspectiva parte de la concepción del método analítico como una actitud de análisis del discurso basada en la contrastación dialéctica entre la teoría y la práctica (Lopera y otros, 2010a). La aplicación del método analítico tiene como consecuencia la formación de una actitud analítica que consta de cuatro procesos que se presentan de forma no lineal: entender, criticar, contrastar e incorporar (Ramírez, 1991; Lopera y otros, 2010a). Entender es captar la forma en que está estructurado y articulado un discurso, así como su sentido y la intención de quien lo emite. Criticar implica comparar sus diferentes partes para poner a prueba su consistencia, examinando posibles contradicciones $\mathrm{o}$ incoherencias internas. También implica compararlo con otros discursos del mismo autor y de otros autores, para examinar su consistencia externa. Contrastar es examinar las consecuencias que un discurso tiene al llevarse a la práctica y su capacidad de predicción de los efectos de la teoría.

La contrastación se realiza mediante experiencias empíricas (hacer realmente lo que dice la teoría) o discursivas (representar sus consecuencias en un ambiente virtual -que puede ser el pensamiento, experimentos mentales, modelos teóricos o modelos computarizados, entre otros). Incorporar es un concepto que indica que durante la vida se van haciendo propios diversos saberes, conocimientos, teorías, generalmente sin analizar, $y$ se van consolidando como pre-juicios que orientan las acciones. Sin embargo, incorporar, en el sentido analítico, implica hacer propios saberes que han sido analizados, pasando de los prejuicios a los pre-supuestos: supuestos previos que se asumen como conjeturas que orientan la existencia, pero que en tanto conjeturas son flexibles $y$ son sometidos a la contrastación con la experiencia. En última instancia, desde esta perspectiva, lo que se incorpora es el método, el cual conforma una actitud analítica en el investigador. 


\section{Método Clínico}

El método científico tiene analítico está el método clínico, el cual es definido por Pasternac (2003) como el estudio en extensión y en profundidad de un sujeto. Según el mismo autor, es lo opuesto al método experimental definido como el estudio de una variable o unas pocas variables en múltiples sujetos en condiciones controladas por el investigador. Para Corral (1994) el método clínico es un método para la resolución de problemas, que tiene la misma estructura del método general de conjeturas y refutaciones propuesto por Popper (1980).

Una característica del método clínico es que se enfoca en el estudio de casos particulares (Pasternac, 2003), la otra es que se basa en indicios (Eco y Sebeok, 1989; Pulice, Manson y Zelis, 2001). La primera característica implica el estudio y la intervención de las situaciones no repetibles, en un ambiente natural, sin modificación intencional del fenómeno por parte del investigador (Piaget, 2008). La segunda implica que el estudio o la intervención se realizan partiendo de conjeturas que surgen de pequeñas huellas, marcas o síntomas, y pretenden develar una situación global que subyace a dichos indicios.

Se puede hablar aquí de un tipo de lógica llamada por Peirce (1955) abductiva, la cual consiste en generar conjeturas a partir de indicios. Luego dichas conjeturas son sometidas a contrastación con la experiencia con el fin de determinar su validez. La intuición es aquí un factor decisivo dado que se aplica naturalmente en aquellas situaciones en las cuales no hay reglas pre-establecidas (casos particulares).

Al igual que el método analítico, el método clínico implica una serie de procesos, pero no hay acuerdo general entre los autores acerca de ellos (Ilizástigui y Rodríguez, 2010; Bercherie,
2009; Dunker, 2011 -citado por Orejuela, Moreno y Salcedo, 2012; Compas y Gotlib, 2003; Godoy, 1996). En general se puede hablar de: observar, diagnosticar, intervenir y pronosticar. En la medicina clásica, observar es captar indicios de la enfermedad con la vista fundamentalmente, pero también con los otros sentidos. Diagnosticar es realizar una clasificación de la enfermedad con el fin de conocer lo que padece el enfermo. Intervenir es poner en marcha la terapéutica apropiada para sanar una enfermedad determinada. Pronosticar, es evaluar el curso futuro de la intervención a la luz de sus efectos.

Es evidente que, desde la anterior perspectiva, el método clínico está enfocado en la enfermedad y no es clara la relación con la organización. Pero existe otra perspectiva: el método clínicoanalítico, que se aplica también en otros ámbitos además del ámbito de la salud, como el derecho (cfr. Correa, 2009; Palermo \& Evans, 2008; Molina, 2008; Correa, 2007; Villarreal y Courtis, 2007), la pedagogía (cfr. Piaget, 2008; Vygotski, 2001; Delval, 2001), la sociología y el trabajo social (cfr. Rodríguez, 2010; De Gaulejac, Rodríguez y Taracena, 2006), la administración y el campo organizacional (cfr. Malvezzi, 2012a, 2012b; Bendassolli, 2011; Bendassolli E Soboll, 2011; Rossi, Mendes, Siqueira e Garcia, 2009; Richardson, 2006; Schvarstein, 2001; Aubert y De Gaulejac, 1993).

Para nosotros, el método clínico se basa en cuatro procesos, no necesariamente lineales: escuchar, formalizar, conjeturar e intervenir. Para estudiar e intervenir una situación singular es importante escuchar el discurso con el fin de analizarlo. No es lo mismo oír que escuchar. Oír es captar emisiones acústicas con el órgano de la audición. En cambio, escuchar es abrirse al interpretar (Ramírez, 2012, ens. 228), con los órganos de los sentidos, el intelecto y las emociones, para entender el discurso. De esta forma, alguien puede oír sin escuchar. 
Formalizar es el proceso de darle forma a lo escuchado, mediante imágenes y palabras, con el fin de discernir qué está sucediendo en el caso estudiado. Conjeturar es crear, proponer posibles explicaciones y alternativas a la situación estudiada, teniendo en cuenta los efectos que podrían tener los diferentes cursos de acción que se pueden tomar, descartando los que se consideren inconvenientes y eligiendo los mejores. Intervenir es llevar a la práctica concreta las conjeturas y cursos de acción determinados evaluando sus efectos en un proceso que implica la posibilidad de revisarlos, modificarlos $\mathrm{o}$ sustituirlos.

Una práctica adicional muy interesante es teorizar sobre el caso y la intervención realizada, con el fin de captar los aciertos y los posibles errores y así consolidar los aciertos y revisar las fallas. Este es un nivel de formalización mayor que puede generar un modelo o una teoría que explique el fenómeno investigado.

Cuando se aplica este método hablamos de una práctica clínica de orientación analítica que definimos como: la práctica profesional de un analítico que utiliza el método clínico en su oficio o trabajo con el propósito de propiciar el cuidado (cura, en el sentido foucaultiano del término) de un sujeto (individual, grupal o colectivo).

Una práctica profesional es aquella que se ejerce como un oficio remunerado bajo las condiciones institucionales definidas en una comunidad determinada. Un analítico es aquella persona que ha incorporado el método analítico como actitud. El cuidado (epimeleia en griego y cura en latín) para Foucault $(2001,1990)$ es la inquietud o preocupación que un sujeto refiere a sí mismo y a la relación que establece consigo y con los otros. Este es el cuidado de sí (cura sui). Un sujeto es en esencia un ser humano (o un conjunto de ellos) que ha pasado de ser un sujetado (de la cultura) y ha tomado cierta distancia de los condicionantes socio-culturales adquiriendo la capacidad de obrar como agente con un cierto grado de consciencia (Lopera y otros, 2010b). El cuidado contribuye al desarrollo humano ya que posibilita la formación de cada sujeto para hacer uso de sus capacidades y posibilidades de una forma responsable. Más adelante se abordará este tema.

Como puede observarse, esta definición habla de una concepción de la clínica que no está relacionada exclusivamente con la salud y la enfermedad, que incluso puede desligarse de dichos conceptos y que puede aplicarse en otros ámbitos, como de hecho se hace.

\section{Organización}

En la teoría administrativa las organizaciones están compuestas de individuos que conforman grupos que actúan para conseguir ciertos fines y objetivos mediante funciones diferenciadas racionalmente coordinadas y dirigidas, y con una cierta continuidad a través del tiempo (Porter, Lawler \& Hackman, 1975). Las organizaciones raramente se establecen como un fin en sí mismas, son instrumentos creados para conseguir unos fines. Dichos instrumentos son considerados, desde la metáfora mecanicista, como dispositivos mecánicos y su existencia está determinada como un vehículo para la realización de diferentes actividades para obtener un fin (Morgan, 2006). En la tradición del liberalismo económico retomado por Hayek (1994), la organización es considerada como un sistema creado y controlado totalmente por el ser humano (taxis, por oposición a cosmos, que es un sistema natural, espontáneo).

Schvarstein (2001, p. 29) plantea que organización "es un concepto cultural, convencional, existente a través de la construcción que de él se hace y que, como toda descripción, existe en el lenguaje". Para este autor, una organización es una construcción social, 
"un conjunto ordenado y estructurado de perceptos, una imagen perceptiva" que se evidencia en establecimientos (tales como escuelas, hospitales, fábricas) atravesados "verticalmente" por diversas instituciones, fenómeno que este autor denomina atravesamiento (Schvarstein, 2001, p. 29; 1998, pp. 293 y ss.). La organización, dice Schvarstein, no siempre se rige de forma pasiva por lo que está ya instituido (la que así lo hace es denominada por él organización-objeto), sino que hay organizaciones que se modifican a partir de la fuerza instituyente (denomina organización-sujeto a las que así lo hacen), en diferentes grados de mayor o menor autonomía. De lo contrario, aclara Schvarstein (2001, 1998) las organizaciones permanecerían inmutables. En los organismos vivos ocurre algo similar. Para Canguilhem, la salud consiste en la capacidad que tiene un organismo de instituir nuevas normas de vida cuando sus condiciones así lo requieran (Canguilhem, 2009; cfr. Lopera, 2016).

\section{La organización como concreción de instituciones}

Para nosotros la distinción de Hayek (1994) entre sistemas tipo taxis y sistemas tipo cosmos difícilmente se sostiene desde una perspectiva científica y filosófica contemporánea (cfr. Popper, 1974; Polanyi, 1967, 2005; Bunge, 2004) y desde la perspectiva de la complejidad (cfr. Morin, 1994). Hayek (1994) imagina un mundo dividido entre dos clases de organizaciones: los organismos $\mathrm{y}$ las organizaciones propiamente dichas. Los organismos o sistemas tipo cosmos son organizaciones "naturales", espontáneas, no planeadas ni creadas por el hombre, tales como los cuerpos de los animales o las instituciones sociales (el lenguaje, el mercado, la salud, la educación, entre otras). Por el contrario, las organizaciones propiamente dichas o sistemas taxis, serían organizaciones creadas y controladas por el ser humano. El problema que encontramos en la propuesta de Hayek es que tal distinción no es apropiada para describir la realidad, ya que todo sistema creado por el hombre posee en mayor o menor medida aspectos que escapan a su voluntad creadora. La posición de Hayek no acepta en dichos sistemas la incidencia del azar: ni de lo indeterminado (azar fuerte), ni de lo no previsible (azar débil) (Mandelbrot, 1996; Cfr. Manrique y Gil, 2013).

En contraste, desde la perspectiva de los estudios organizacionales (De La Rosa y Contreras, 2007), la organización conceptualizada por Hayek (1994), descrita por la teoría administrativa (Porter, Lawler \& Hackman, 1975) y propia del liberalismo económico, ha sido catalogada como instrumental (Vergara, 2005; Chanlat, 2002; Polanyi, 1992) teniendo en cuenta que su objetivo básico es la generación de utilidades a partir de recursos materiales, financieros $y$ humanos. Para los estudios organizacionales, la organización es un ente complejo que posee aspectos predecibles y controlables y otros emergentes, impredecibles $y$, en consecuencia, incontrolables (De La Rosa y Contreras, 2007; cfr. Schvarstein, 2001; Etkin, 2005; Lopera y otros, 2010a).

Desde nuestra perspectiva, una organización es la concreción de instituciones (Manrique, Lopera, Pérez, Ramírez y Henao, 2016). Basándonos en Malinowski (1984), Schvarstein (2001), Lopera y otros (2010a) y Ramírez (2011), definiremos institución como un discurso fundante, esto es, un discurso que funda $\mathrm{y}$ fundamenta valores, hábitos y prácticas. Dicha concreción de instituciones se realiza en tres perspectivas: formal, material y práctica. La concreción formal se realiza mediante la expresión de las instituciones en palabras (de forma oral o escrita) o fórmulas. Son ejemplo de este tipo de concreción ciertas creencias básicas de una persona, las tradiciones 
orales de un pueblo, las políticas de una empresa, las leyes de un país. $\mathrm{La}$ concreción material de las instituciones se refiere a la expresión materializada de las mismas. Son ejemplo de dicha concreción la construcción de herramientas, la adecuación de espacios para estudiar, trabajar, descansar, la construcción de máquinas, entre otros. Finalmente, la concreción práctica de las instituciones es la incorporación que realiza cada una de las personas de los valores, hábitos, estilos de vida que la sociedad le proporciona, los cuáles se manifiestan en sus prácticas.

Como ejemplo de la concreción de instituciones, podemos pensar en una organización educativa, atravesada por discursos fundantes como la educación (pedagogía constructivista con énfasis en humanidades), la economía (una organización pública sin ánimo de lucro), la salud (modelo basado en la prevención), la religión (organización confesional católica), entre. Dichas instituciones se formalizan en diversos aspectos de la organización educativa como su visión, su misión, sus políticas. Se materializan en los espacios diseñados como aulas de clase, la capilla y símbolos religiosos, la biblioteca, las canchas deportivas, el servicio médico. Se ponen en práctica en las clases participativas, el tipo de evaluación que realizan los docentes, la celebración de las fiestas religiosas, las jornadas de prevención de la enfermedad y promoción de la salud, entro otros aspectos.

\section{El hombre como animal verbal}

Si aceptamos la definición de organización como concreción de instituciones, hemos de aceptar que el ser humano es una especie de organización (Manrique y otros, 2016). En efecto, el ser humano al nacer va incorporando (concretando) una serie de instituciones sociales que le posibilitan adquirir su condición de ser humano propiamente dicho. La conjunción entre el organismo biológico humano y la cultura, mediatizada por las instituciones sociales y el lenguaje, se realiza mediante un proceso de estructuración (socialización, culturización, formación), el cual genera como resultado una estructura (alma, personalidad, psiquismo) y a partir de esta se dan unos efectos (conductas) (Lopera y otros, 2010b).

En consecuencia, estamos de acuerdo con Ramírez (2012) quien plantea que el ser humano es un animal verbal. El verbo es la esencia del hombre (varón o mujer). Verbo es la traducción latina de logos, palabra griega de difícil traducción que tiene múltiples significados, de los cuales nos interesa uno: articulación. Para Aristóteles (Étic. Nic., 1098a-1098b) la esencia del ser humano es el logos. Con frecuencia se traduce esta idea diciendo que el ser humano es un animal racional, linguístico, simbólico, semántico, político. Se enfatiza con cada una de estas definiciones el aspecto de la articulación propuesto por Ramírez (2012) que incluye a las otras. Lo propio del ser humano es la capacidad de articular su vida mediante el lenguaje (Polanyi, 2005). De allí que la verbación, esto es, la articulación de imágenes en palabras, sea la acción más consustancial del ser humano Ramírez (2012).

De acuerdo con Zapata (1995; cfr. Pérez y Lopera, 2016), cuando el ser humano verbaliza, puede lograr tres efectos: simbolización, socialización y responsabilización. La simbolización es representar un objeto mediante otro, que puede ser algo material, una imagen o una palabra; simbolizar es el principio de la formalización. La socialización es compartir consensualmente lo simbolizado; es el principio del pacto social que posibilita que los seres humanos vivamos en comunidad. La responsabilización es un proceso que implica tomar consciencia y responder de manera reflexiva, ante una circunstancia determinada, teniendo en cuenta que el 
otro puede demandar que se asuma lo dicho; esto es lo que diferencia nuestras respuestas de las de otros seres como los animales, las plantas y los minerales quienes responden primaria o elementalmente (cfr. Ramírez, 2012, ens. 14; Manrique y Gil, 2013).

$\mathrm{La}$ responsabilización supone que el ser humano es libre de elegir entre dos o más opciones, es decir, posee libre albedrío. A su vez éste solo es posible si se parte de una conceptualización que le dé cabida al azar: lo indeterminado y lo impredecible, dado que, si todo estuviera predeterminado, no habría posibilidad de elección (Ramírez, 2012, ens. 14; Manrique y Gil, 2013; Taleb, 2006).

$$
\text { Consideramos que una }
$$

organización empresarial puede tener una estructura análoga a la de un sujeto individual (una persona) y la llamamos sujeto plural (Manrique y otros, 2016). En efecto, para su conformación es necesaria la conjunción de aspectos materiales (seres humanos, infraestructura) con aspectos discursivos (instituciones mediatizadas por el lenguaje) y pasar por un proceso de estructuración, cuyo resultado es una estructura, la cual genera una serie de efectos (comportamientos). Asimismo, consideramos que un grupo puede entenderse como un sujeto grupal (Schvarstein, 2001) dado que posee las mismas características generales de un sujeto individual y de un sujeto colectivo. La utilidad de estos conceptos radica en que podemos comprender que el sujeto grupal $\mathrm{y}$ el sujeto colectivo son amplificaciones del sujeto individual, esto es, no hay una discontinuidad radical entre unos y otros, lo cual posibilita pensar en términos de interdependencia entre ellos, en el sentido de un nosotros, que se opone a la tendencia individualista predominante en los modelos socio-económicos instrumentales.

\section{Desarrollo humano}

Desde nuestra perspectiva, dado que el ser humano es un animal verbal, lo fundamental para su desarrollo es ejercitar dicha capacidad. Por lo tanto, el desarrollo humano estará fundamentado en las condiciones que hagan posible la verbalización como acción que posibilita la toma de decisiones analizadas. De acuerdo con Sen (2000; cfr. Saldarriaga, 2010), las condiciones institucionales en un contexto socio-político son fundamentales para el desarrollo humano. Los regímenes totalitarios, en los cuales está prohibido expresar opiniones diferentes a las oficialmente reconocidas, coartan la libertad, impidiendo que la verbalización se produzca. Asimismo, los contextos neoliberales en los que se privilegia la competencia a ultranza y se asume el dinero como valor fundamental por encima de la cooperación, pueden conducir a la inequidad en la distribución de los recursos, generando con ello la imposibilidad para una parte de la población de un país de acceder a los servicios institucionales básicos fundamentales para su supervivencia $\mathrm{y}$ desarrollo como son alimentación, salud, ciudadanía, educación, empleo, entre otros.

Nosotros agregamos a lo planteado por Sen (2000) que además de unas condiciones externas propicias para el desarrollo humano, son fundamentales condiciones psicológicas internas a los sujetos. Vemos a menudo que algunas personas, a pesar de contar con posibilidades externas para acceder a la educación, no lo hacen debido a barreras psicológicas o no deciden hacer uso de las oportunidades reales que ofrecen las instituciones.

Es aquí donde consideramos importante contribuir con espacios analíticos a la formación para la toma de decisiones (Manrique y De Castro, en revisión; Manrique, 2017). Como se mencionó anteriormente, se analizan los 
discursos con el fin de comprenderlos, contribuyendo a realizar elecciones adecuadas, esto es, a tomar decisiones convenientes, de acuerdo con criterios de desarrollo humano. A pesar de que todos tomamos decisiones continuamente, tomar decisiones adecuadas en diferentes ámbitos (personal, familiar, académico, laboral, político) no es algo sencillo y requiere un proceso de formación.

Este ámbito de la toma de decisiones es de sumo interés en la actualidad (World Bank, 2015). Específicamente el debate se presenta en torno a la toma de decisiones basada en estadísticas (generalización, mecanización) o basada en la intuición (caso por caso: juicios clínicos). De acuerdo con Grove (2005), Meehl estudió la toma de decisiones basada en estadísticas, llegando a la conclusión de que es más eficaz, aunque con excepciones (cuando se presentan casos atípicos que rompen con las regularidades). En cambio, Klein (2008, 1991) ha estudiado la toma de decisiones en ambientes naturales en momentos que no es posible basarse en estadísticas o reglas pre-establecidas (como por ejemplo bomberos, militares, psicólogos clínicos, operadores bursátiles, equipos de trabajo), llegando a la conclusión de que es posible generar estrategias para mejorar la intuición y la toma de decisiones mediante el análisis y la formalización de los procesos implicados. Consideramos que es en este ámbito (investigación y formación de la intuición y toma de decisiones) en el cual el método clínicoanalítico toma toda su relevancia, pues los procedimientos estadísticos son de difícil aplicación en estos contextos ya que son imposibles de operar en momentos y casos concretos $\mathrm{y}$, cuando es posible aplicarlos, requieren demasiado tiempo e información. No obstante, esto sólo es posible si en la organización existe una filosofía de gestión que lo sustente y una gestión humana que lo ponga en práctica.

\section{Gestión humana y filosofía de gestión}

La gestión humana se ha entendido tradicionalmente como una de las áreas que conforman la organización empresarial. Sin embargo, a lo largo de la investigación hemos encontrado que organización es un concepto más amplio que el de empresa (López, 2005), pues encontramos organizaciones sin ánimo de lucro, políticas, deportivas, de salud, educativas, entre otras que mal hacen si se rigen como empresas poniendo el lucro como aspecto principal por encima de su razón social (Cortina, 1994; Etkin, 2007; Schvarstein, 2001). Cuando esto ocurre, argumenta Etkin (2007; cfr.), la organización se pervierte y pierde su razón de ser deslegitimándose ante la sociedad. El concepto de empresa entendido como una clase de organización productiva o de servicios, privada y con ánimo de lucro (López, 2005), ha de subordinarse al de organización en el sentido amplio aquí planteado. Entonces caben en el concepto de organización un ser humano, una comunidad, una organización empresarial $\mathrm{y}$, en última instancia, la sociedad completa, que no es otra cosa que una supra-organización que agrupa las otras organizaciones.

Desde esta perspectiva, gestión humana es un concepto amplio que no se restringe a un área específica de una organización empresarial encargada de realizar procesos de integración, organización, desarrollo y auditoría (Chiavenato, 2007). Si revisamos el significado general de la palabra gestión, el concepto viene del latín gestio-onis: gestión, de gerere: llevar a cabo, acción de administrar o hacer diligencias (Corripio, 1979). Por su parte, Chanlat (2006) plantea que a menudo la gestión ha sido considerada por diversos autores como un conjunto de prácticas y actividades fundadas sobre cierto número de principios que apuntan a una finalidad: la búsqueda de la eficacia. Esta idea consensual posibilita pensar que la gestión 
humana es, entonces, una actividad que realiza todo ser humano con el fin de lograr un deseo con respecto a otros seres humanos. Diciéndolo, en otros términos, la gestión humana se refiere a las relaciones de poder y las estrategias que se ponen en marcha con el propósito de mediar entre (y articular) los intereses propios y los intereses de otros (Henao, 2016). De esta forma, la gestión humana cumple una función mediadora en las relaciones y tensiones socio-laborales que se establecen entre los seres humanos, el trabajo y la organización. Para abordar este tema, nos centraremos en la gestión humana en la organización empresarial.

Ahora bien, para llevar a cabo el proceso de mediación es necesario contar con un marco de referencia conceptual, llamado filosofía de gestión. Este permite realizar reflexiones sobre la razón de ser y dirección de la organización (misión y visión), y las pautas de comportamiento esperadas de los sujetos que la componen, conteniendo las premisas fundamentales de las que se derivan las políticas de la organización. Dichas premisas se refieren a la concepción del ser humano, la organización, el trabajo y el mundo. La filosofía de gestión está constituida por aquellos principios básicos (valores) que orientan la acción de los sujetos en la organización. Existen dos tipos de filosofía de gestión: disociadora e integradora (Etkin, 2007).

La filosofía de gestión disociadora opone las variables económicas a las socio-culturales, teniendo como principio fundamental una concepción del ser humano basada en la idea del homo economicus. Se trata de un ser humano que actúa de forma egoísta pensando en sus propios intereses personales y cuya principal finalidad es maximizar sus ingresos económicos (Etkin, 2007). Es denominado tonto racional, debido a que parece ser que actúa ingenuamente de forma totalmente calculadora (Sen, 2000; Schwartz, 2011; Saldarriaga, 2010).
Este tipo de hombre es una pieza del engranaje de la organización entendida metafóricamente como una máquina con todas las piezas mecánicas que deben estar en su lugar cumpliendo adecuadamente su función que es previsible y totalmente calculada. $\mathrm{O}$ como un organismo vivo que contiene a su vez otros organismos vivos, los cuales responden a motivaciones intrínsecas (psicológicas, emocionales) y extrínsecas (premios y castigos). $\mathrm{O}$, en el mejor de los casos, como un cerebro, esto es, un computador súper desarrollado (la misma máquina, pero más compleja) compuesto por neuronas o microchips que trabajan en red a partir de una estructura holográfica: en el todo está la parte y en la parte está el todo (Morgan, 2006; Muñoz, 2002). Dicha organización es el ámbito en el que se realiza el trabajo, actividad netamente instrumental cuyo medio son una serie de recursos, entre ellos el recurso humano, y el fin es el aumento de la riqueza (fundamentalmente capital económico).

La filosofía de gestión disociadora parte de una concepción del mundo profundamente religiosa: el mundo está organizado perfectamente de manera que existen una serie de reglas naturales que controlan el mercado y no deben ser perturbadas por la intervención del ser humano a riesgo de caer es un régimen totalitario (Hayek, 1994; cfr. Polanyi, 1992, para una posición diferente). Lo único que el ser humano debe hacer es seguir la ley de la libre competencia en la cual ganarán los más fuertes o los que son bendecidos por la gracia de Dios (Aubert y De Gaulejac, 1993; Weber, 1991).

Desde la perspectiva de la filosofía de gestión integradora, se aboga por una actitud fundada, responsable y comprometida con la calidad de vida de las organizaciones, integrando las variables económicas, sociales y culturales (Etkin, 2007). El aumento de la riqueza es importante, pero no debe lograrse a cualquier costo. El ser humano es aquí un fin en sí mismo y la riqueza es 
un medio, ya que se concibe como un ser libre con capacidad de desarrollo, fundamentalmente basado en la cooperación (Saldarriaga, 2010; Sen, 2000). En este sentido, la organización es una expresión del ser humano construida como un medio eficaz para satisfacer sus necesidades y las de la sociedad en general. Es por ello que una organización empresarial adquiere legitimidad (Cortina, 1994; Muñoz, 2002).

A su vez, el trabajo es una actividad mediante la cual el ser humano satisface necesidades de supervivencia $\mathrm{y}$ superiores (espirituales), incrementa la riqueza entendida en un sentido amplio (no solo referido a lo financiero), pero además es un espacio de construcción de identidad, de actualización de potencialidades (Aubert y De Gaulejac, 1993; Clot, en Bendassolli e Soboll, 2011). Desde la perspectiva de Ramírez (2012) y Lopera (2016), esto sería parte de un despliegue armónico del ser.

De igual manera, desde la filosofía integradora, consideramos que el mundo es un espacio de tensión entre diversos intereses que requieren de una mediación a través de la intervención del ser humano quien ha de buscar la equidad, esto es, que cada vez sean menos las personas que tengan una restricción para usar su libre albedrío, para elegir de forma conveniente ante los sucesos aleatorios que se presentan diariamente. Es un mundo en construcción que puede ser modificado en cierto grado por los seres humanos, quienes también han de contar con lo inmodificable y con el azar. Es un mundo que le apuesta a la cooperación dada la interdependencia del ser humano, que posibilita pensarnos como un nosotros (Ramírez, 2012) en el cual propendemos a integrar los diversos intereses personales, grupales y colectivos, basados en un principio de co-responsabilidad.

Cuando se cuenta con una filosofía de gestión integradora, se genera un ambiente más propicio para plantear una gestión humana de orientación analítica que promueva la responsabilización y el desarrollo humano de los actores que interactúan en y con la organización. Esta orientación propicia la realización de trabajos grupales basados en espacios de verbalización y análisis del discurso con los diferentes sujetos, en los cuales se logran simbolizar aspectos que hasta ese momento han permanecido en forma tácita generando en ocasiones malestar, dificultades o re-procesos. Asimismo, en ellos se logran socializar símbolos, aspectos culturales, saberes desconocidos, prácticas; se crea un saber compartido y se establecen pactos, se redefinen otros y se aclaran malos entendidos. Adicionalmente, en dichos espacios se generan procesos de responsabilización en la medida en que un aumento en la articulación de cada ser humano complejiza su identidad, aclara aspectos propios y del entorno, aprende a tomar mejores decisiones y genera confianza. Mediante el actuar en común, la toma de decisiones en conjunto $y$ el habla compartida pueden llegar a desarrollarse las facultades $y$ potencialidades que posibilitan tanto la conducción autónoma de la propia vida como la vida con otros (Saldarriaga, 2010).

\section{Clínica analítica de las organizaciones}

Hemos considerado que hay una relación de continuidad e interdependencia entre los sujetos individuales, que pueden expresarse como sujetos grupales y colectivos. Dicha relación de continuidad se establece debido a que los procesos de estructuración del sujeto en estos tres niveles son similares y están basados en lo que constituye la esencia del ser humano, a saber, el $\operatorname{logos}$ en tanto verbo: articulación, verbación (Ramírez, 2012; cfr. Polanyi, 2005). Dicha articulación se presenta en la elaboración de discursos, expresados en palabras o no, en un ámbito individual, grupal y colectivo. Para que el 
ser humano desarrolle sus posibilidades inherentes de elección y toma de decisiones es necesario un proceso de verbalización y análisis que es posible promover mediante filosofías o principios fundamentales de las organizaciones que conduzcan a la apertura de espacios de diálogo y participación, que es el punto que desarrollaremos en este apartado.

Para abordar este tema tomaremos como ejemplo algunas organizaciones empresariales. Una pregunta recurrente que se hacen las organizaciones se refiere al papel de los líderes de equipos de trabajo en el desarrollo de cada empleado (incluidos ellos). Uno de los aspectos fundamentales de nuestra propuesta fue la relación entre el trabajo con grupos en la organización, los elementos del método clínico que se utilizan en el día a día y el desarrollo humano (Ramírez y Manrique, 2016).

Consideramos que son importantes los espacios de verbalización, aunque se enfatiza en la importancia de manejarlos con prudencia debido a los riesgos inherentes que existen al "liberar la palabra". En el análisis de las entrevistas y el grupo de discusión (que abordamos en otro lugar: Pérez y Lopera, y 2016; Ramírez y Manrique, 2016), encontramos testimonios de la eficacia del trabajo con grupos $\mathrm{y}$, en general, aquellos espacios que propenden a la verbalización en términos de simbolización, socialización y responsabilización. Además, encontramos que eran valorados por los entrevistados como aspectos que contribuyen a su desarrollo, manifestando sentirse reconocidos como seres humanos cuando son escuchados $\mathrm{y}$ sus opiniones son tenidas en cuenta (aunque no necesariamente llevadas a la práctica).

Muchos de los profesionales entrevistados en el marco del proyecto de investigación, utilizan técnicas clínicas en su quehacer laboral. Otros lamentan que algunos líderes de la organización carezcan de estas herramientas, especialmente a la hora de coordinar los equipos de trabajo y realizar una mediación en los conflictos que se presentan entre los diferentes sujetos de la organización (individual, grupal, colectivo). Por último, otros entrevistados hablan sobre procesos que se están llevando a cabo con el fin de capacitar a los líderes en aspectos que denominamos clínicos (aunque ellos no lo llaman de esta manera) porque corresponden a la conceptualización que hacemos de dicho concepto.

Recordemos que consideramos que son aspectos clínico-analíticos aquellos que están enfocados al estudio e intervención de situaciones concretas tomando cada caso como un caso único y teniendo como finalidad el cuidado del sujeto (Manrique y otros, 2016). En este sentido, el conflicto (o la situación a analizar) que se puede presentar en un grupo $\mathrm{X}$, de la empresa $\mathrm{Y}$, es único $\mathrm{e}$ irrepetible, lo cual quiere decir que no es posible tratarlo bajo modelos estandarizados, estadísticos (aunque pueda ser interesante en un momento dado hacer comparaciones con casos anteriores o de otras empresas: por ejemplo, la decisión que se tomó en otro momento puede ser inadecuada para este caso concreto).

Por eso es fundamental el trabajo con grupos mediante el cual se generen espacios en los que se propicie la expresión, escucha y análisis de los discursos de los sujetos. Dicho trabajo tiene como propósito general transmitir la actitud analítica, lo cual procura el cuidado, en el sentido foucaultiano de epimeleia heautou, de inquietud de sí (Foucault, 2001). Esto implica el análisis de la posición asumida por cada sujeto respecto a un caso concreto, la responsabilidad que le compete y las decisiones que debe tomar. Ello, sobra decirlo, se logra en la medida en que haya un ambiente de reconocimiento por parte del otro (empezando por los directivos), lo cual conduce al respeto.

El método clínico-analítico aplicado en el trabajo con grupos es 
entonces un método para el abordaje organizacional de la tensión entre los intereses de los sujetos: individuales, grupales y colectivos, tensión que es inherente a la condición de seres humanos. Es conveniente porque se enfoca en cada caso concreto, apuesta por el respeto hacia el otro y es eficaz en tanto posibilita el análisis de la situación por parte de los sujetos involucrados. Además, propende a la formación en la toma de decisiones adecuadas para la construcción de un nosotros que privilegia la convivencia armónica y la consecución de los intereses de cada uno sin desconocer las necesidades del grupo y la organización, lo cual es factible mediante la cooperación, por oposición al individualismo.

\section{Conclusiones}

La aplicación del método clínico tradicionalmente ha sido asociada a contextos terapéuticos en torno al concepto de enfermedad. Esto ha restringido la posibilidad de ser considerado en otros campos ocupacionales $\mathrm{y}$, especialmente, en los escenarios organizacionales. Esta propuesta conceptual articula el método clínico con el analítico y propende al cuidado del sujeto individual, grupal $\mathrm{y}$ colectivo en las organizaciones. Para esto consideramos que es necesario contar con una filosofía de gestión integradora, que fundamente las prácticas de gestión humana, teniendo como premisas fundamentales una concepción del hombre, el trabajo, la organización y el mundo, que favorezca el despliegue de las potencialidades y el desarrollo humano organizacional.

Es importante precisar las limitaciones de la propuesta dado su alcance y características. Por ello no se detallan los aspectos prácticos para su implementación, ni se especifican los elementos hallados en aquellas empresas que ya han llevado a cabo algunas aplicaciones del método clínico-analítico (aunque no lo denominen asî). El énfasis en el análisis del discurso en el trabajo individual y grupal por medio de espacios conversacionales, es conveniente para fortalecer las dinámicas grupales y la cohesión social. Consideramos que con el desarrollo de esta propuesta teórica se formalizan algunos constructos que pueden dar cabida a nuevos desarrollos teórico-empíricos en diversos campos profesionales, en los que el método clínico-analítico aún no se ha aplicado. Si bien es cierto que algunos autores cuestionan este tipo de propuestas por considerar que existe un riesgo de construir sujetados, antes que propiciar la subjetivación (cfr. García y Carvajal, 2006; Aubert y De Gaulejac, 1993), es importante plantear alternativas para el desarrollo humano vía la responsabilización, evitando justamente la cosificación y homogenización de los sujetos en las organizaciones.

\section{Referências}

Aristóteles. (1970). Ética a Nicómaco. Madrid: Instituto de estudios Políticos.

Aubert, N. y De Gaulejac, V. (1993). El coste de la excelencia. Barcelona: Paidós.

Bedoya, M. y Schnitter, M. (2012). Las rutas de emergencia de la psicología clínica y sus impertinencias. Revista de Psicología de la Universidad de Antioquia, v. 2, n. 3, p. 23-39.

Bendassolli, P. (2011). Mal-estar no trabalho: do sofrimento ao poder de agir. Revista Mal-estar e Subjetividade, v. 10, n. 1, p. 63-98.

Bendassolli, P. e Soboll, L. (orgs.). (2011). Clínicas do Trabalho. Novas 
perspectivas para compreensão do trabalho na actualidade. São Paulo, Atlas.

Bercherie, P. (2009). Los fundamentos de la clínica. Historia y estructura del saber psiquiátrico. Buenos Aires: Manantial.

Bermúdez, H. (2013). Sociología Clínica y Psicodinámica del Trabajo en el estudio del placer y el sufrimiento en el trabajo cotidiano. Teuken Bidikay, 4, 153-180.

Bunge, M. (2004). Emergencia y convergencia. Novedad cualitativa y unidad del conocimiento. Barcelona: Gedisa.

Chanlat, J. (2002). Ciencias Sociales y Administración. Medellín: Fondo Editorial Universidad EAFIT.

Chiavenato, I. (2007). Administración de recursos humanos. El capital humano de las organizaciones. México D. F.: McGraw-Hill.

Compas, B. y GOTLIB, I. (2003). Introducción a la psicología clínica. México D. F: Mc Graw Hill.

Corral, C. (1994). El razonamiento médico. Los fundamentos lógicos del método clínico. Madrid: Ediciones Díaz de Santos.

Correa, A. (Comp.). (2009). La enseñanza clínica del derecho. Colombia: Fundación Universitaria Luis Amigó; Fundación Universitaria Tecnológico Comfenalco, Cartagena.

Correa, L. (2007). Investigación pura y aplicada a través de una clínica jurídica. Revista Universidad de Medellín, v. 42, n. 83, p. 67-78.

Corripio, F. (1979). Diccionario etimológico general de la lengua castellana. Barcelona: Bruguera.
Cortina, A. (1994). Ética de la empresa. Madrid. Editorial Trotta.

De Gaulejag, V; Rodríguez, S. y Taracena, E. (2006). Historia de vida. Psicoanálisis y sociología clínica. Querétaro: Universidad Autónoma de Querétaro.

De La Rosa, A. y Contreras, J. (2007). El partido político: entre la ciencia política y los estudios organizacionales. Polis: Investigación y análisis sociopolítico y psicosocial, v. 3, n. 2, 17-67.

Delval, J. (2001). Descubrir el pensamiento de los niños: introducción a la práctica del método clínico. Barcelona: Paidós.

Eco, U. y Sebeok, T. (1989). El signo de los tres. Barcelona: Lumen.

Etkin, J. (2005). Gestión de la complejidad en las organizaciones. Buenos Aires: Granica.

Foladori, H. (2008). La intervención institucional. Hacia una clínica de las instituciones. Santiago: Editorial ARCIS.

Foucault, M. (2001). Hermenéutica del sujeto. Buenos Aires: Fondo de Cultura Económica.

Foucault, M. (1990). Tecnologías del yo y otros textos afines. Barcelona: Paidós.

Fritz, J. (1991). The Contributions of Clinical Sociology in Health Care Settings. Health Sociology, 9(1), 1529.

García, C. y Carvajal, L. (2006). Tecnologías empresariales del yo: la construcción de sujetos laborales en el contexto del trabajo inmaterial. Universitas psychologica, v. 6, n. 1, p. 49-58. 
Gaulejac, V. de, Rodríguez, S. y Taracena, E. (2006). Historia de vida. Psicoanálisis y sociología clínica. Querétaro: Universidad Autónoma de Querétaro.

Gigerenzer, G. (2007). Gut feelings. The intelligence of the unconscious. USA: Viking.

Godoy, A. (1996). Toma de decisiones y juicio clínico. Una aproximación psicológica. Madrid: Pirámide.

Grove, W. (2005). Clinical versus statistical prediction: the contribution of Paul E. Meehl. Journal of clinical psychology, v. 61, n. 10, p. 12331243.

Hayek, F. (1994). Derecho, legislación y libertad. Una nueva formulación de los principios liberales de la justicia y de la economía política. Vol. I. Normas y orden. Madrid: Unión Editorial.

Hein, S. \& Austin, W. (2001). Empirical and hermeneutic approaches to phenomenological research in psychology: a comparison. Psychological methods, v. 6, n. 1, p. 3-17.

Henao, C. (2016). Teoría y práctica de la mediación. Una aplicación desde la perspectiva de Gilbert Simondon y el método analítico en las organizaciones. Tesis de doctorado en administración, Universidad EAFIT, Medellín, Colombia.

Ilizástigui, F. y Rodríguez, L. (2010). El método clínico. Revista Electrónica de las Ciencias Médicas en Cienfuegos, Medisur; Suplemento "El método clínico", v. 8, n. 5, p. 2-11.

Jaeckel, M. (1991). Clinical Sociology. Teaching Sociology, 19(1), 96-102.
Kahneman, D. \& Klein, G. (2009). Conditions for intuitive expertise: A failure to disagree. American Psychologist, v. 64, n. 6, p. 515-526.

Klein, G. (2008). Naturalistic decisionmaking. Journal of human factors and ergonomics society, v. 50, n. 3, p. 456-460.

Klein, G. (1991). Naturalistic decisionmaking. Human system IAC GATEWAY. v. 11, n. 3.

Lapassade, G. y Lourau, R. (1973). Claves de sociología. Barcelona: Laia.

Laverty, S. (2003). Hermeneutic phenomenology and phenomenology: a comparison and methodological considerations. International journal of qualitative methods, v. 2, n. 3.

Lopera, J. (2016). Salud psíquica y sabiduría práctica. Bogotá: San Pablo.

Lopera, J., Ramírez, C., Zuluaga, M. у Ortiz, J. (2010a). El método analítico como método natural. Nómadas Revista de la Universidad Complutense de Madrid (25), p. 325 353.

Lopera, J; Manrique, H; Zuluaga, M. y Ortiz, J. (2010b). El objeto de la psicología: el alma como cultura encarnada. Medellín: Universidad de Antioquia.

López, F. (2005). "Administración", "organización" (y "empresa"): un intento de acotación semántica. Revista Universidad EAFIT, n. 137, 9-18.

Malinowski, B. (1984). Una teoría científica de la cultura. Madrid, Sarpe.

Malvezzi, S. (2012a). El desempeño en la era de las empresas-red: Desafíos para 
la investigación de la subjetividad. En: MALVEZZI, S; OREJUELA, J; CHIUZI, R; VESGA, J. y RIASCOS, W. Gramáticas actuales de la relación hombre - trabajo. Propuestas de lectura. Cali: Editorial Bonaventuriana.

Malvezzi, S. O (2012b). trabalho tras felicidade? En: Revista Marketing industrial, v. 16, n 56, p. 66-75.

Mandelbrot, B. (1996). Del azar benigno al azar salvaje. Revista Investigación y Ciencia, n. 243.

Manrique, H. (2017). Intuición y deliberación en la toma de decisiones: la experiencia de los decisores. Tesis de doctorado en psicología. Universidad del Norte. Barranquilla, Colombia.

Manrique, H. (2008). Saber y conocimiento: una aproximación plural. Acta Colombiana de psicología, v. 11, n. 2, p. 89-100.

Manrique, H. y De Castro, A. (En revisión). Toma de decisiones: intuición y deliberación en la experiencia de los decisores.

Manrique, H. y Gil, L. (2013). Azar, libertad y responsabilidad. Aportes para una práctica psicológica. Pensamiento psicológico, v. 11, n. 2, p. 143-155.

Manrique, H., Lopera, I., Pérez, J., Ramírez, V. y Henao, C. (2016). Clínica analítica de las organizaciones. Santafé de Bogotá: Editorial San Pablo.

Molina, C. (2008). Fundamentos teóricos y metodológicos del método clínico de enseñanza del derecho. Revista Facultad de Derecho y Ciencias Políticas, v. 38, n. 108, p. 187-213.
Morgan, G. (2006). Images of organization. USA: Sage publications, 2006.

Morin, E. (1994). Introducción al pensamiento complejo. España. Madrid: Gedisa.

Muñoz, R. (2002). Paradigmas organizacionales y gestión humana. Por una nueva ética de la relación laboral. Revista Universidad EAFIT, n. 127 , p. 9-17.

Nonaka, I. y TEECE, D. (ed.) (2001). Manging industrial knowledge. Creation, transfer and utilization. SAGE publications.

Orejuela, J. (2018). Clínica del trabajo. El malestar subjetivo derivado de la fragmentación laboral. Bogotá: San Pablo.

Orejuela, J; Moreno, M. y Salcedo, M. (comp.) (2012). Abordajes psicoanalíticos a inquietudes sobre la subjetividad. Santiago de Cali: Bonaventuriana.

Palermo, J. \& Evans, A. (2008). Almost there. Empirical insights into clinical method and ethics courses in climbing the hill towards lawyers professionalism. Griffith Law Review, v. 17, n. 1, p. $252-284$.

Pasternac, M. (2003). El método experimental y el método clínico en psicología. En: Braunstein, $\mathrm{N}$; Pasternac, M; Benedito, G. y Saal, F. Psicología: ideología y ciencia. México: Siglo XXI, p. 127-155.

Pérez, J. y Lopera, I. (2016). Gestión humana de orientación analítica: un camino para la responsabilización. RAE-Revista de Administração de Empresas, v.56, n. 1, p. 101-113. 
Pierce, C. (1955). Abduction and induction. En: Philosophical writings of Peirce. New York: Dover Publications, p. 150-156.

Piaget, J. (2008). La representación del mundo en el niño. Madrid: Morata, 2008 .

Polanyi, K. (1992). La gran transformación. Los orígenes políticos y económicos de nuestro tiempo. México D. F.: Fondo de Cultura Económica.

Polanyi, M. (2005). Articulation. In: Personal knowledge. Towards a postcritical philosophy. London: Taylor \& Francis e-Library, p. 70-139.

Polanyi, M. (1967). The tacit dimension. USA, Anchor Books.

Popper, K. (1980). La lógica de la investigación científica. Madrid: Editorial Tecnos.

Popper, K. (1974). Sobre nubes y relojes. En: Conocimiento objetivo. Madrid: Tecnos, p. 193-235.

Porter, L.; Lawler, E.; Hackman, J. (1975). Behavior in organizations. New York: McGraw-Hill, 1975.

Pulice, G; Manson, F. y Zelis, O. (2001). La práctica de la investigación en relación al pensamiento mágico, la conjetura, el paradigma indiciario y la ciencia moderna. Revista Cinta de Moebio, n. 12, p. 79-101.

RAE (2001). Diccionario de la lengua española. Vigésima segunda edición. [En línea] Disponible en: http $/ /$ rae.es/ Consultado 17/10/2013.

Ramírez, C. (2012). La vida como un juego existencial: ensayitos. Medellín: Fondo Editorial Universidad EAFIT.
Ramírez, C. (2011). Análisis de las instituciones. Inédito.

Ramírez, C. (1991). El método científico en el psicoanálisis. Revista Universidad de Antioquia, Medellín, v. 60 , n. 224 , p. $35-41,1991$.

Ramírez, C.; Lopera, J.; Zuluaga, M. y Ortiz, J. (2017). El método analítico. Bogotá: San Pablo.

Ramírez, V. y Manrique, H. (2016). Método clínico y trabajo analítico con grupos en el ámbito organizacional (pp. 415-436). En: Orejuela, J., Andrade, V. y Villamizar, M. Psicología de las organizaciones y del trabajo. Apuestas de investigación II. Cali: Editorial Bonaventuriana.

Richardson, M. (2006). The people management clinic: Answers to Your Most Frequently Asked Questions. London: Thorogood Publishing Ltd.

Rodríguez, M. (2010). La intervención social como objeto de análisis clínico. Portularia, v. 10, n. 1, 73-84.

Ross, K; Klein, G; Thunholm, P; Schmitt, J. \& Baxter, H. (2004). The recognition-primed decision model. Military review, v. 74, n. 4, p. 6-10.

Rossi, E; Mendes, A; Siqueira, M. y García, J. (2009). Sedução e servidão em um caso de LER/DORT: diálogo entre a pscodinâmica do trabalho e a sociología clínica. Psicología política, v. 9 , n. 18, p. 313-330.

Ryle, G. (1949/2005). El concepto de lo mental. España, Paidós.

Saldarriaga, A. (2010). El sujeto activo: antropología política en Amartya Sen. Eidos: Revista de filosofía de la Universidad del Norte, n. 3, p. 54-75. 
Schvarstein, L. (2001). Psicología social de las organizaciones. Buenos Aires: Paidós.

Schvarstein, L. (1998). Diseño de organizaciones. Tensiones $y$ paradojas. Buenos Aires: Paidós.

Schwartz, B. (2011). Practical wisdom and organizations. Research in organizational behavior, n. 31, p. 323.

Sen, A. (2000). Desarrollo y libertad. Barcelona: Planeta.

Taleb, N. (2006). ¿Existe la suerte? Engañados por el azar. Madrid: Thomson-Paraninfo.

Vergara, J. (2005). La concepción de Hayek del estado de derecho y la crítica de Hinkelammert. Polis, n. 10, p. 1-12.

Villarreal, M. y Courtis, C. (coordinadores) (2007). Enseñanza clínica del derecho. Una alternativa a los métodos tradicionales de formación de abogados. México D. F.: Sans Serif Editores.

World Bank. (2015). World Development Report 2015: Mind, Society, and Behavior. Washington, DC: World Bank.

Vygotski, L. (2001). Pensamiento y lenguaje. En: Obras escogidas. Vol. II. Madrid: Machado.

Weber, M. (1991). La ética protestante y el espíritu del capitalismo. Tlahuapan. Puebla: Premia.

Wirth, L. (1931). Clinical Sociology. American Journal of Sociology, 37(1), 49-66.

Zapata, L. (1995). La verbalización. De la curación por la palabra al método psicoanalítico. Tesis de pregrado en psicología. Medellín: Universidad de Antioquia. Sin publicar. 